\title{
Cutting Degree of Meanders
}

\author{
A. Panayotopoulos ${ }^{1}$ and Panayiotis Vlamos $^{2}$ \\ 1 University of Piraeus \\ 80 Karaoli \& Dimitriou Str, 18534 Piraeus, Greece \\ antonios@unipi.gr \\ 2 Department of Informatics, Ionian University \\ Plateia Tsirigoti 7, 49100 Corfu, Greece \\ vlamos@ionio.gr
}

\begin{abstract}
We study the cutting problems of meanders using 2-Motzkin words. These words uniquely define elevated peakless Motzkin paths, which under specific conditions correspond to meanders. A procedure for the determination of the set of meanders with a given sequence of cutting degrees, or with a given cutting degree, is presented by using proper conditions.
\end{abstract}

Keywords: Dyck path, Grand Dyck path, 2-Motzkin word, elevated peakless Motzkin path.

\section{Introduction}

The cutting problem of meanders has been introduced by the authors in 9 . Using meandric polygons, we have also enumerated the set of meanders with given numbers of cuttings, i.e., the numbers of arcs of the meandric curve lying above and below the horizontal line at a given point.

In the present paper, we complete the cutting problem of meanders. At first, we represent a meander or meandric system using Motzkin words, and we specify the subset of meandric Motzkin words, through a necessary and sufficient condition. Additionally, we determine the matching nested sets of a meandric Motzkin word. Afterwards, we establish a bijection between meandric Motzkin words and elevated peakless Motzkin paths. We calculate the cardinality of this class, as well as the number of these paths, passing through a given point. Finally, we confront the two main cutting problems of meanders, as we determine the set of meanders with a given sequence of cutting degrees, or with a given cutting degree.

The two cutting problems can be considered as data-mining problems, since we extract the subset of Motzkin words which are meandric, or the subset of Motzkin paths which are peakless and elevated. Specifically in the field of Bioinformatics, the already mentioned problems have many facets in relation with the M-folding problem and RNA prediction problem, based on the fact that closed RNA structures can be viewed as mathematical objects obtained by abstracting topologically non-relevant properties of planar folding of single-stranded nucleic

L. Iliadis et al. (Eds.): AIAI 2012 Workshops, IFIP AICT 382, pp. 480-489, 2012.

(c) IFIP International Federation for Information Processing 2012 
acids. In the literature, many approximation data-mining algorithms had been constructed, concerning the enumeration of sequential terms of RNA secondary structures, through the bijection with alternative representations such as energy models, plane trees and Motzkin numbers [11, $k$-noncrossing set of partitions [3], set of nested arcs [3], Motzkin paths and Dyck paths [5].

The following definitions and notation refer to notions that are necessary for the development of the paper. A set $S$ of disjoint pairs of $[2 n]$ such that $\bigcup_{\{a, b\} \in S}\{a, b\}=[2 n]$ and for any $\{a, b\},\{c, d\}$ we never have $a<c<b<d$, is called nested set of pairs on $[2 n]$. We denote the set of all nested sets of pairs on $[2 n]$ by $N_{2 n}$. Two nested sets $S_{1}, S_{2} \in N_{2 n}$ define a permutation $\sigma$ on [2n], such that $\sigma(2 i-1)=j$ iff $\{2 i-1, j\} \in S_{1}$ and $\sigma(2 i)=j$ iff $\{2 i, j\} \in S_{2}$, for every $i \in[n]$. The sets $S_{1}, S_{2}$ are $k$-matching if and only if $\sigma$ has $k$ cycles. In the case where $k=1, S_{1}, S_{2}$ are simply called matching.

A word $v \in\{a, \bar{a}\}^{*}$ is called Dyck word if $|v|_{a}=|v|_{\bar{a}}$ and for every factorization $v=p q$ we have $|p|_{a} \geq|p|_{\bar{a}}$ where $|v|_{a},|p|_{a}$ (resp. $|v|_{\bar{a}},|p|_{\bar{a}}$ ) denote the number of occurrences of $a$ (resp. $\bar{a})$ in the words $v$ and $p$. A word $v \in\{a, \bar{a}\}^{*}$, such that $|v|_{a}=|v|_{\bar{a}}$ is called Grand Dyck word. We denote by $D_{2 n}$ the set of all Dyck words of length $2 n$ and it is well known that the cardinality of $D_{2 n}$ is equal to the $n$-th Catalan number $C_{n}=\frac{1}{n+1}\left(\begin{array}{c}2 n \\ n\end{array}\right)$.

\section{Meanders}

A (closed) meander of order $n$ is a closed self avoiding curve, crossing a horizontal line $2 n$ times [4. We denote by $M_{2 n}$ the set of meanders of order $n$ [].

We can extend the definition of meanders to meandric systems with $k$ components, by allowing configurations with $k$ disconnected meanders [4].

In this paper, every meander of $M_{2 n}$ is represented by a word $w \in\{a, \bar{a}, b, \bar{b}\}^{*}$, which is called meandric Motzkin word [8]. For example, the meander of Fig. 1$]$ corresponds to the word $w=a a \bar{b} b$ a $b \bar{a} \bar{a} \bar{b} \bar{a}$.

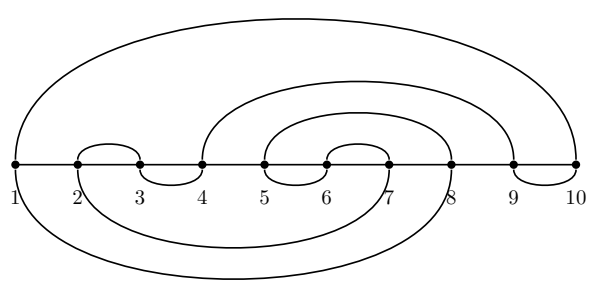

Fig. 1. A meander with $w=a a \bar{b} b a b \bar{a} \bar{a} \bar{b} \bar{a}$

In order to formally introduce the meandric Motzkin words, following [8], we recall that for every pair $v=v_{1} v_{2} \cdots v_{\ell}$ and $v^{\prime}=v_{1}^{\prime} v_{2}^{\prime} \cdots v_{\ell}^{\prime}$ of words in $\{a, \bar{a}\}^{*}$ of length $\ell$, we define $v \circ v^{\prime}$ to be the word $w=w_{1} w_{2} \cdots w_{\ell} \in\{a, \bar{a}, b, \bar{b}\}^{*}$, with

$$
w_{i}=v_{i} \circ v_{i}^{\prime}, i \in[\ell], \quad \text { where } a \circ a=a, \bar{a} \circ \bar{a}=\bar{a}, a \circ \bar{a}=b, \bar{a} \circ a=\bar{b} .
$$


Note that the words $v$ and $v^{\prime}$ can be uniquely recovered from $w$ using the following relations

$$
v_{i}=\left\{\begin{array}{ll}
a, & \text { if } w_{i}=a \text { or } b, \\
\bar{a}, & \text { if } w_{i}=\bar{a} \text { or } \bar{b},
\end{array} \quad \text { and } \quad v_{i}^{\prime}= \begin{cases}a, & \text { if } w_{i}=a \text { or } \bar{b} \\
\bar{a}, & \text { if } w_{i}=\bar{a} \text { or } b\end{cases}\right.
$$

for $i \in[\ell]$, and we call $v, v^{\prime}$ associates of $w$.

We define the set of the meandric Motzkin words of length $2 n$ as the set

$$
\hat{W}_{2 n}=\left\{w: w=v \circ v^{\prime}, v, v^{\prime} \in D_{2 n}\right\} .
$$

Notice that from the word $v \circ v^{\prime}$ we immediately obtain the word $v^{\prime} \circ v$, by interchanging the letters $b$ and $\bar{b}$. So, in order to generate the set $\hat{W}_{2 n}$ it is actually enough to construct half of its elements.

The set $\hat{W}_{2 n}$ can be partitioned into the classes $W_{2 n}^{k}, k \in[n]$, where $W_{2 n}=$ $W_{2 n}^{1}$. Each class $W_{2 n}^{k}$ contains the meandric Motzkin words with $v, v^{\prime} \in D_{2 n}$, such that their corresponding nested sets are $k$-matching.

It has been proved in [8] that there exists a bijection between the sets $M_{2 n}$ and $W_{2 n}$. According to this bijection, every meander $\mu$ can be considered as an ordered pair $(U, L)$ of nested sets of pairs, where $U$ (resp. $L$ ) is defined by the arcs of the meandric curve above (resp. below) the horizontal line. Since $U, L$ are encoded by two Dyck words, say $v$ and $v^{\prime}$, then the meandric Motzkin word $w=v \circ v^{\prime}$ encodes the meander $\mu$.

In [8, it was also proved (see Proposition 2.1) that every word $w$ of $\hat{W}_{2 n}$ is a 2-Motzkin word of $\{a, \bar{a}, b, \bar{b}\}^{*}$, with $|w|_{b}=|w|_{\bar{b}}$. More specifically, if $w \in \hat{W}_{2 n}$, then by deleting the letters $b, \bar{b}$ (resp. $a, \bar{a}$ ) in $w$, a Dyck word (resp. Grand Dyck word) is obtained, or equivalently, we have that $|w|_{a}=|w|_{\bar{a}}, \quad|w|_{b}=$ $|w|_{\bar{b}}$ and $\quad|p|_{a} \geq|p|_{\bar{a}}$, for every factorization $w=p q$. Consequently, every word $w \in \hat{W}_{2 n}$ can be considered as the shuffle of a Dyck word in $\{a, \bar{a}\}^{*}$ and a Grand Dyck word in $\{b, \bar{b}\}^{*}$, where the first and last letter of $w$ are $a$ and $\bar{a}$ respectively. Obviously, not every word $w$ satisfying the above conditions encodes a meander, or a meandric system. As an example, consider the word $a \bar{b} \bar{b} b b \bar{a}$. The next result states the necessary and sufficient condition for a word to be a meandric Motzkin word.

Proposition 1. For a word $w \in\{a, \bar{a}, b, \bar{b}\}^{*}$ of length $2 n$, we have that $w \in \hat{W}_{2 n}$ if and only if $|w|_{a}-|w|_{\bar{a}}=|w|_{b}-|w|_{\bar{b}}=0$ and $|p|_{a}-|p|_{\bar{a}} \geq\left.|| p\right|_{b}-|p|_{\bar{b}} \mid$, for every factorization $w=p q$.

Proof. Let $w \in\{a, \bar{a}, b, \bar{b}\}^{*}$ and let $w=p q$ be a factorization of $w$. Then, the associates $v, v^{\prime}$ of $w$, such that $w=v \circ v^{\prime}$, are uniquely defined and, furthermore, the factorization of $w$ uniquely defines the factorizations $v=r s$ and $v^{\prime}=r^{\prime} s^{\prime}$, such that $p=r \circ r^{\prime}$ and $q=s \circ s^{\prime}$. By definition, we have the following relations

$$
|r|_{a}=|p|_{a}+|p|_{b}, \quad|r|_{\bar{a}}=|p|_{\bar{a}}+|p|_{\bar{b}}, \quad\left|r^{\prime}\right|_{a}=|p|_{a}+|p|_{\bar{b}}, \quad\left|r^{\prime}\right|_{\bar{a}}=|p|_{\bar{a}}+|p|_{b},
$$

which lead to

$$
|r|_{a}-|r|_{\bar{a}}=|p|_{a}-|p|_{\bar{a}}+|p|_{b}-|p|_{\bar{b}} \quad \text { and } \quad\left|r^{\prime}\right|_{a}-\left|r^{\prime}\right|_{\bar{a}}=|p|_{a}-|p|_{\bar{a}}-\left(|p|_{b}-|p|_{\bar{b}}\right)
$$


Note that the above relations also hold, when we set $p=w$, and in this case we have $r=v$ and $r^{\prime}=v^{\prime}$.

If $w \in \hat{W}_{2 n}$, then $v, v^{\prime} \in D_{2 n}$, so that $|r|_{a}-|r|_{\bar{a}} \geq 0,\left|r^{\prime}\right|_{a}-\left|r^{\prime}\right|_{\bar{a}} \geq 0$ and $|v|_{a}-|v|_{\bar{a}}=\left|v^{\prime}\right|_{a}-\left|v^{\prime}\right|_{\bar{a}}=0$. In this case, from relations (10), we have that $|p|_{a}-$ $|p|_{\bar{a}} \geq|p|_{\bar{b}}-|p|_{b}$ and $|p|_{a}-|p|_{\bar{a}} \geq|p|_{b}-|p|_{\bar{b}}$, or equivalently $|p|_{a}-|p|_{\bar{a}} \geq\left.|| p\right|_{b}-|p|_{\bar{b}} \mid$, while applying relations (1), for $p=w$, we obtain that $|w|_{a}-|w|_{\bar{a}}=|w|_{\bar{b}}-|w|_{b}$ and $|w|_{a}-|w|_{\bar{a}}=|w|_{b}-|w|_{\bar{b}}$, or equivalently $|w|_{a}-|w|_{\bar{a}}=|w|_{b}-|w|_{\bar{b}}=0$.

Conversely, if $|w|_{a}-|w|_{\bar{a}}=|w|_{b}-|w|_{\bar{b}}=0$ and $|p|_{a}-|p|_{\bar{a}} \geq\left.|| p\right|_{b}-|p|_{\bar{b}} \mid$, then relations (10) imply that $|r|_{a}-|r|_{\bar{a}} \geq 0$ and $\left|r^{\prime}\right|_{a}-\left|r^{\prime}\right|_{\bar{a}} \geq 0$, while applying relations (11) for $p=w$, we obtain that $|v|_{a}-|v|_{\bar{a}}=\left|v^{\prime}\right|_{a}-\left|v^{\prime}\right|_{\bar{a}}=0$. From the above, we deduce that $v, v^{\prime} \in D_{2 n}$, so that $w \in \hat{W}_{2 n}$.

The process of determining the matching nested sets $U, L$ that correspond to a meander $w \in W_{2 n}$ can be simplified by the following easily verified proposition:

Proposition 2. Let $U, L$ be the matching nested sets that correspond to a meander $w \in W_{2 n}$. Then, every pair of $U$ (resp. L) corresponds to a subsequence of $w$ having one of the following forms: $a \bar{a}, a \bar{b}, b \bar{a}, b \bar{b}$ (resp. $a \bar{a}, a b, \bar{b} \bar{a}, \bar{b} b$ ).

According to Proposition 2, the short pairs of $U$ (i.e., pairs of the form $\{i, i+1\}$ ) are immediately determined by identifying the positions of the occurrences of the digrams $a \bar{a}, a \bar{b}, b \bar{a}, b \bar{b}$ in $w$. Then, by deleting these digrams from $w$, we obtain a new word $w^{\prime} \in\{a, \bar{a}, b, \bar{b}\}^{*}$. We repeat the same procedure for $w^{\prime}$, until all pairs are determined. The pairs of $L$ are obtained analogously.

For example, for the meander $w=a a \bar{b} b a b \bar{a} \bar{a} \bar{b} \bar{a}$ of Fig. 1, the nested set $U$ is obtained as follows:

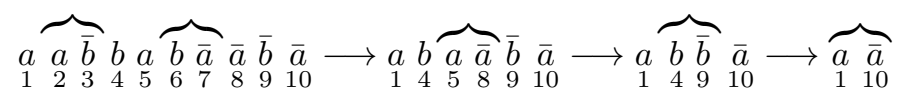

Thus, $U=\{\{1,10\},\{2,3\},\{4,9\},\{5,8\},\{6,7\}\}$. Analogously, we obtain that $L=\{\{1,8\},\{2,7\},\{3,4\},\{5,6\},\{9,10\}\}$.

\section{Examples}

1. The meandric Motzkin word $w=a b b b \bar{b} a \bar{b} \bar{a} \bar{b} \bar{a}$, does not belong to the set $\hat{W}_{10}$. This is verified by the following table:

\begin{tabular}{|c|c|c|c|c|}
\hline & \begin{tabular}{|l|l|l|l|l|l|l|l}
$a$ & $b$ & $b$
\end{tabular} & $b \bar{b}$ & & \\
\hline$|p|_{a}-|p|_{\bar{a}}$ & 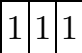 & & & \\
\hline$|p|_{b}-|p|_{\bar{b}} \mid$ & 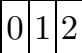 & & & \\
\hline
\end{tabular}

2. The meandric Motzkin word $w=a b \bar{b} a b \quad \bar{a} \bar{b} b \bar{b} \bar{a}$, belongs to the set $w \in W_{10}$. This is verified by the following table

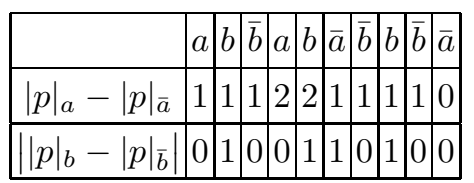


and by the fact that its corresponding nested sets

$U=\{\{1,10\},\{2,3\},\{4,7\},\{5,6\},\{8,9\}\}, L=\{\{1,2\},\{3,6\},\{4,5\},\{7,8\},\{9,10\}\}$,

which are obtained using Proposition 2 are matching.

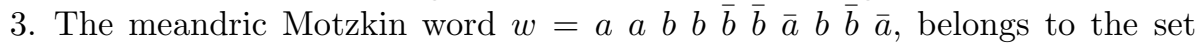
$w \in W_{10}^{2}$. This is verified by the following table

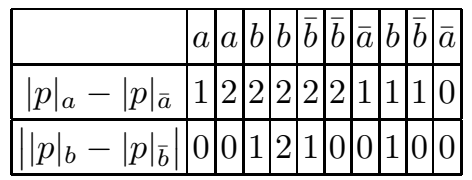

and by the fact that its corresponding nested sets

$U=\{\{1,10\},\{2,7\},\{3,6\},\{4,5\},\{8,9\}\}, L=\{\{1,4\},\{2,3\},\{5,8\},\{6,7\},\{9,10\}\}$,

which are obtained using Proposition 2, are 2-matching.

\section{Motzkin Paths}

We recall that a Motzkin path (resp. 2-Motzkin path) is a lattice path in the first quadrant beginning at the origin, ending at the $x$-axis and consisting of the steps $a=(1,1)$ as the up-step, $\bar{a}=(1,-1)$ as the down-step and $b=(1,0)$ as the horizontal step (resp. $a, \bar{a}$ and two kinds of horizontal steps denoted by $b$ and $\bar{b}$ ). Every Motzkin path (resp. 2-Motzkin path) is encoded by its sequence of steps, i.e., a word $y \in\{a, \bar{a}, b\}^{*}$ (resp. $y \in\{a, \bar{a}, b, \bar{b}\}^{*}$ ). The set of all Motzkin paths (resp. 2-Motzkin paths) of length $n$ is enumerated by the $n$-th Motzkin number $\mathrm{M}_{n}=\sum_{k \geq 0}\left(\begin{array}{c}n \\ 2 k\end{array}\right) C_{k}$ [2], (resp. the number $C_{n+1}$ [1]).

An elevated peakless Motzkin path, is a Motzkin path that never touches the $x$-axis except at its first and last points and it has no peaks (i.e., no occurrences $a \bar{a})$. For example, the path of Fig. 2 is an elevated peakless Motzkin path of length 10 with $y=a a b b a b \bar{a} \bar{a} b \bar{a}$. We denote by $P_{2 n}$ the set of all elevated peakless Motzkin paths of length $2 n$.

Given that (see [10, A004148) the number of peakless Motzkin paths of length

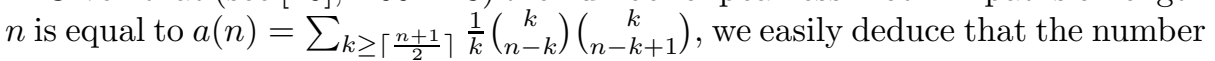
of paths of $P_{2 n}$ is equal to $a(2 n-2)$. Thus, we have the following result.

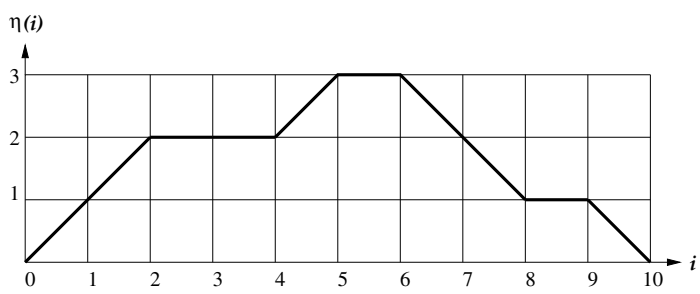

Fig. 2. The elevated peakless Motzkin path with $y=a a b b a b \bar{a} \bar{a} b \bar{a}$ 
Proposition 3. The cardinality of $P_{2 n}$ is given by

$$
\sum_{k \geq\left\lceil\frac{2 n-1}{2}\right\rceil} \frac{1}{k}\left(\begin{array}{c}
k \\
2 n-k-2
\end{array}\right)\left(\begin{array}{c}
k \\
2 n-k-1
\end{array}\right) .
$$

A list of terms of the above sequence are presented in [10], A203019.

The set $P_{2 n}$ can also be encoded in $\{0,1,2, \ldots, n\}$ by the heights $\eta(i)$ of the vertices $(i, \eta(i)), i=0,1, \ldots, 2 n$ of the path. For the heights $\eta(i)$ we have the following properties:

(i) $\eta(0)=\eta(2 n)=0, \eta(1)=\eta(2 n-1)=1$.

(ii) $1 \leq \eta(i) \leq \min \{i, 2 n-i\}, i \in[2 n-1]$.

(iii) $|\eta(i)-\eta(i-1)|=0$ or $1, i \in[2 n]$.

(iv) There exists no $i \in[2 n-2]$, such that $\eta(i)=\eta(i+2)=\eta(i+1)-1$.

Indeed, the first property holds since each path of $P_{2 n}$ begins at $(0,0)$, ends at $(2 n, 0)$ and it is elevated. The second property is deduced by the fact that the difference of the number of occurrences of $a$ 's and $\bar{a}$ 's at the $i$-step is at most $i$, if $i \leq n$ or $2 n-i$, if $i \geq n$. The third property is obvious since at each step we add $+1,-1$ or 0 to the height of the previous point. The last property is an immediate consequence of the fact that the path is peakless.

By omitting the initial and final terms $\eta(0)=\eta(2 n)=0$, we introduce the finite sequences $\eta=(\eta(i))_{i \in[2 n-1]}$, and their set will be denoted by $H_{2 n-1}$.

For example, for the path of Fig. 2, we obtain the sequence

$$
\eta=122233211 \text {. }
$$

In general, to every path $y \in P_{2 n}$ corresponds a sequence $\eta \in H_{2 n-1}$ with

$\eta(1)=\eta(2 n-1)=1 \quad$ and $\quad \eta(i)= \begin{cases}\eta(i-1)+1, & \text { if } y_{i}=a \\ \eta(i-1), & \text { if } y_{i}=b, \quad 2 \leq i \leq 2 n-2 . \\ \eta(i-1)-1, & \text { if } y_{i}=\bar{a},\end{cases}$

The converse also holds, since to every sequence $\eta \in H_{2 n-1}$ corresponds a path $y \in P_{2 n}$ with

$$
y_{1}=a, y_{2 n}=\bar{a} \quad \text { and } \quad y_{i}= \begin{cases}a, & \text { if } \eta(i)>\eta(i-1), \\ b, & \text { if } \eta(i)=\eta(i-1), \quad 2 \leq i \leq 2 n-1 \\ \bar{a}, & \text { if } \eta(i)<\eta(i-1),\end{cases}
$$

So, we have arrived at the following proposition.

Proposition 4. There exists a bijection between the sets $P_{2 n}$ and $H_{2 n-1}$.

From the above bijection between the sets $P_{2 n}$ and $H_{2 n-1}$, we deduce that the cardinality of the set $H_{2 n-1}$ is given by the formula of Proposition 3 .

The paths of $P_{2 n}$ can be obtained by their corresponding sequences of $H_{2 n-1}$ following the above properties of the heights $\eta(i)$.

This is done with the aid of a suitable tree of height $2 n-1$, in which each vertex is labeled by an integer of $[n]$, so that: 
(i) if a vertex labeled by $\xi$ belongs to the $e^{\text {th }}$ level, then $\xi \leq \min \{e, 2 n-e\}$,

(ii) to every vertex with label $\xi$ correspond at most three children, with labels $\xi-1, \xi, \xi+1$, on $[n]$.

(iii) at every path of the tree, we can not find three consecutive vertices with labels $\xi, \xi+1, \xi$ on $[n]$.

Our next effort concerns the number $a_{i, k}$ of paths of $P_{2 n}$ passing through the point $(i, k)$. We already know (see [10, A097724) that the number of left factors of peakless Motzkin paths, having length $n$ and endpoint height $k$ is equal to $T(n, k)=(k+1) \sum_{j \geq\left\lceil\frac{n-k+1}{2}\right\rceil} \frac{1}{j}\left(\begin{array}{c}j \\ n-k-j\end{array}\right)\left(\begin{array}{c}j+k \\ n+1-j\end{array}\right)$.

The number of left factors for the paths of $P_{2 n}$ starting from $(0,0)$ and ending at $(i, k)$ is given by $T(i-1, k-1)=k \sum_{j \geq\left\lceil\frac{i-k+1}{2}\right\rceil} \frac{1}{j}\left(\begin{array}{c}j \\ i-k-j\end{array}\right)\left(\begin{array}{c}j+k-1 \\ i-j\end{array}\right)$.

Furthermore, the number of right factors for the paths of $P_{2 n}$ starting from $(i, k)$ and ending at $(2 n, 0)$ is equal to the number of left factors for the paths of $P_{2 n}$ starting from $(0,0)$ and ending at $(2 n-i, k)$, i.e., $T(2 n-i-1, k-1)=$ $k \sum_{j \geq\left\lceil\frac{2 n-i-k+1}{2}\right\rceil} \frac{1}{j}\left(\begin{array}{c}j \\ 2 n-i-k-j\end{array}\right)\left(\begin{array}{c}j+k-1 \\ 2 n-i-j\end{array}\right)$. Thus, we have the following result.

Proposition 5. The number of paths of $P_{2 n}$ passing through the point $(i, k)$ is given by the product $k^{2} \sum_{j \geq\left\lceil\frac{i-k+1}{2}\right\rceil} \frac{1}{j}\left(\begin{array}{c}j \\ i-k-j\end{array}\right)\left(\begin{array}{c}j+k-1 \\ i-j\end{array}\right) \sum_{j \geq\left\lceil\frac{2 n-i-k+1}{2}\right\rceil} \frac{1}{j}\left(\begin{array}{c}j \\ 2 n-i-k-j\end{array}\right)\left(\begin{array}{c}j+k-1 \\ 2 n-i-j\end{array}\right)$.

The paths of $P_{2 n}$ passing through the point $(i, k)$ are obtained by the subtree of the tree of $H_{2 n-1}$ with $\eta(i)=k$.

We denote by $P_{2 n}^{(2)}$ the set of elevated peakless 2-Motzkin paths $y$ of length $2 n$, with $|y|_{b}=|y|_{\bar{b}}$. Since the length of every path $w \in P_{2 n}$ and the number $|w|_{a}+|w|_{\bar{a}}$ are even, we conclude that $|w|_{b}$ is also even. Therefore, every path $y \in P_{2 n}^{(2)}$, with $|y|_{b}=|y|_{\bar{b}}=\nu$, is uniquely obtained from a path $w \in P_{2 n}$, with $|w|_{b}=2 \nu$, by converting half of the $b$ steps of $w$ into $\bar{b}$ steps. Conversely, $w \in P_{2 n}$ generates $\left(\begin{array}{c}2 \nu \\ \nu\end{array}\right)$ paths of $P_{2 n}^{(2)}$, which cannot be generated by any other path of $P_{2 n}$.

Furthermore, the number of peakless Motzkin paths of length $2 n$ with $k a$ steps and $2 \nu$ horizontal steps is given by the formula (sequence $A 089732$ in [10]) $T(2 n, n-\nu)=\left(\begin{array}{c}2 n-k \\ k\end{array}\right)\left(\begin{array}{c}2 n-k \\ k+1\end{array}\right) \frac{1}{2 n-k}=\left(\begin{array}{c}n+\nu \\ 2 \nu\end{array}\right)\left(\begin{array}{c}n+\nu \\ 2 \nu-1\end{array}\right) \frac{1}{n+\nu}$, where $T(2 n, n-\nu)$ is also the number of paths in $P_{2 n}$ with $2 \nu$ horizontal steps, for $n, \nu>0$.

Finally, since $P_{2 n}$ is partitioned with respect to the number $2 \nu$ of $b$ steps, where $1 \leq \nu \leq n$, using the previous formula, we have the following result.

Proposition 6. The cardinality of $P_{2 n}^{(2)}$ is given by $\sum_{\nu=1}^{n}\left(\begin{array}{c}2 \nu \\ \nu\end{array}\right)\left(\begin{array}{c}n+\nu \\ 2 \nu\end{array}\right)\left(\begin{array}{c}n+\nu \\ 2 \nu-1\end{array}\right) \frac{1}{n+\nu}$.

\section{Cutting Problem}

For every meander of $M_{2 n}$ and for any $i \in[2 n-1]$ we consider the vertical line passing through the middle point of the segment $(i, i+1)$, which we call as the $i$-line 9]. The number of arcs of the meandric curve, which are intersected by the $i$-line is called cutting degree $\epsilon(i)$ of the meander at $i$. 


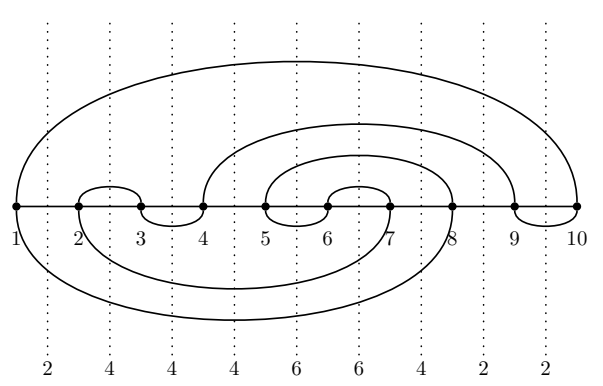

Fig. 3. A meander with $\epsilon=244466422$

For example, for the meander of Fig. 1, we have $\epsilon=244466422$ (see Fig. 33). The cutting degrees for a meander $w \in W_{2 n}$ are obviously deduced by

$$
\epsilon(1)=\epsilon(2 n-1)=2 \text { and } \epsilon(i)= \begin{cases}\epsilon(i-1)+2, & \text { if } w_{i}=a \\ \epsilon(i-1), & \text { if } w_{i}=b \text { or } \bar{b} \\ \epsilon(i-1)-2, & \text { if } w_{i}=\bar{a}\end{cases}
$$

for $i=2,3, \ldots, 2 n-2$.

We denote by $E_{2 n-1}$ the set of the sequences $\epsilon=(\epsilon(i))_{i \in[2 n-1]}$ satisfying

$$
\epsilon(1)=\epsilon(2 n-1)=2 \text { and }|\epsilon(i)-\epsilon(i+1)| \in\{0,2,-2\}, i \in[2 n-2] .
$$

From the previous, we have that

1. To every meander $w \in W_{2 n}$ corresponds a unique finite sequence $\epsilon \in E_{2 n-1}$ but obviously, the converse does not hold.

2. The sequences $\epsilon$ and $\eta$, corresponding to a meander of $M_{2 n}$, satisfy $\epsilon(i)=$ $2 \eta(i), i \in[2 n-1]$.

3. For the cutting degree, the following properties hold

(i) $2 \leq \epsilon(i) \leq 2 \min \{i, 2 n-i\}, i \in[2 n-1]$.

(ii) $|\epsilon(i+1)-\epsilon(i)| \in\{0,2\}, i \in[2 n-1]$.

(iii) There exists no $i \in[2 n-2]$, such that $\epsilon(i)=\epsilon(i+2)=\epsilon(i+1)-2$.

The main cutting problems of meanders are to determine:

a) The subset $M_{2 n}(\epsilon)$ of $M_{2 n}$ with given sequence of cutting degrees $\epsilon$.

b) The subset $M_{2 n}(i, \epsilon(i))$ of $M_{2 n}$ with given cutting degree $\epsilon(i)$ at $i$.

For the first problem, i.e. to determine the set $W_{2 n}(\epsilon)$ we have that if $\epsilon$ is deduced by a given meander then $W_{2 n}(\epsilon) \neq \emptyset$, while if $\epsilon$ is deduced by a given $\eta \in H_{2 n-1}$ then $W_{2 n}(\epsilon)$ could be empty.

So we start by finding the Motzkin word $y \in P_{2 n}$ which corresponds to the sequence $\eta \in H_{2 n-1}$, where $\eta(i)=\frac{\epsilon(i)}{2}$, for $i \in[2 n-1]$.

From $y$ we determine all the 2-Motzkin words $\omega \in\{a, \bar{a}, b, \bar{b}\}^{*}$ with the following properties:

The letter $h$ is replaced by the letters $b, \bar{b}$ such that $|\omega|_{b}=|\omega|_{\bar{b}}$. 
Obviously, if $|y|_{b}=2 \nu$ there exist $\left(\begin{array}{c}2 \nu \\ \nu\end{array}\right)$ such words $\omega$. Those words can be obtained, if we properly place the $b$ 's and $b$ 's in the positions of the $b$ 's, with the help of a binary tree $B_{2 \nu}$ of height $2 \nu$. In this tree, each left child is labeled by $b$ and each right child is labeled by $\bar{b}$, given that the equal number of $b$ 's and $\bar{b}$ 's is $\nu$. Thus, the number of possible meanders belongs to the set $\left\{0,2,4, \ldots,\left(\begin{array}{c}2 \nu \\ \nu\end{array}\right)\right\}$. We notice that the paths of the right subtree of $B_{2 \nu}$ can be produced by replacing the $b$ 's with $\bar{b}$ 's at the paths of the left subtree.

For example, for the Motzkin word $y=a a b b a b \bar{a} \bar{a} b \bar{a}$ of Fig. 2, we have

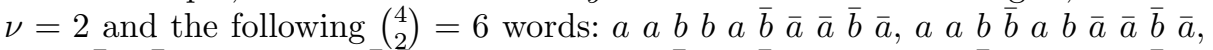
$a a b \bar{b} a \bar{b} \bar{a} \bar{a} b \bar{a}, a a \bar{b} b a b \bar{a} \bar{a} b \bar{a}, a a \bar{b} b a \bar{b} \bar{a} \bar{a} b \bar{a}, a a \bar{b} b a b \bar{a} \bar{a} \bar{b} \bar{a}$ which are obtained with the aid of the tree $B_{4}$.

According to the above, the problem can be confronted by the following procedure:

Step 1. Find the Motzkin word $y$ which corresponds to a given sequence $\eta \in$ $H_{2 n-1}$.

Step 2. Find the tree $B_{2 \nu}$, where $\nu=\frac{1}{2}|y|_{b}$.

Step 3. Find, using $y$ and $B_{2 \nu}$, the 2-Motzkin words $\omega=\omega_{1} \omega_{2} \cdots \omega_{2 n}$ of $\{a, \bar{a}, b, \bar{b}\}^{*}$ for which:

(i) $\omega_{i}=\left\{\begin{array}{ll}a, & \text { if } y_{i}=u, \\ b \text { or } \bar{b}, & \text { if } y_{i}=h, \\ \bar{a}, & \text { if } y_{i}=d,\end{array} \quad\right.$ for $i \in[2 n] . \quad$ (ii) $|\omega|_{b}=|\omega|_{\bar{b}}=\nu$.

Step 4. Find the associate words $r, r^{\prime}$ for each $\omega$ and check if there are Dyck words.

Step 5. Find from the remaining $\omega$, the ones that satisfy the matching property for the corresponding nested sets.

Examples. The following examples illustrate the above procedure.

1) For $\eta=111112211$ we have $y=a b b b b a b \bar{a} b \bar{a}$ and $\nu=3$. From the left subtree of $B_{6}$, we derive $\frac{1}{2}\left(\begin{array}{l}6 \\ 3\end{array}\right)=10$ words.

We can easily observe that none of these words satisfies the condition of the fourth step. Hence, for $\epsilon=222224422$, we have that $W_{10}(\epsilon)=\emptyset$.

2) For $\eta=111221111$ we have that $y=a b b a b \bar{a} b b b \bar{a}$ and $\nu=3$, hence from the left subtree of $B_{6}$, we derive 10 words, only four of them satisfying the conditions of the fourth and fifth step.

Hence, for $\epsilon=222442222$, we have that

$$
\begin{aligned}
& W_{10}(\epsilon)=\{a b \bar{b} a b \bar{a} \bar{b} b \bar{b} \bar{a}, a b \bar{b} a b \bar{a} \bar{b} \bar{b} b \bar{a}, a b \bar{b} a \bar{b} \bar{a} b b \bar{b} \bar{a}, \\
& a b \bar{b} a \bar{b} \bar{a} b \bar{b} b \bar{a}, a \bar{b} b a \bar{b} \bar{a} b \bar{b} b \bar{a}, a \bar{b} b a \bar{b} \bar{a} b b \bar{b} \bar{a}, \\
& a \bar{b} b a b \bar{a} \bar{b} \bar{b} b \bar{a}, a \bar{b} b a b \bar{a} \bar{b} b \bar{b} \bar{a}\} \text {. }
\end{aligned}
$$

3) For the meander of Fig. 11we have $\eta=122233211, y=a$ a $b$ ba $b \bar{a} \bar{a} b \bar{a}$, and $\nu=2$, hence from the left subtree of $B_{4}$, we derive $\frac{1}{2}\left(\begin{array}{l}4 \\ 2\end{array}\right)=3$ words. We can easily observe that all these words satisfy the conditions of the fourth and fifth steps. Hence, for $\epsilon=244466422$, we have that

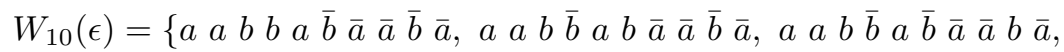

$$
\begin{aligned}
& a a \bar{b} \bar{b} \text { a } b \bar{a} \bar{a} b \bar{a}, a a \bar{b} b a \bar{b} \bar{a} \bar{a} b \bar{a}, a a \bar{b} b a b \bar{a} \bar{a} \bar{b} \bar{a}\} \text {. }
\end{aligned}
$$


We will now solve the second problem, i.e., we will determine the elements of the set $W_{2 n}(i, \epsilon(i))$.

For the existence of such meanders, the conditions that $\epsilon(i)$ is even and $2 \leq$ $\epsilon(i) \leq 2 \min \{i, 2 n-i\}$, for $i=2,3, \ldots, 2 n-2$, must hold.

If the cutting degree at $i$ for a meander is $\epsilon(i)$, then its corresponding path of $P_{2 n}$ passes through the point $(i, \eta(i))=\left(i, \frac{\epsilon(i)}{2}\right)$.

It is natural to find the paths of $P_{2 n}$ passing through the point $(i, \eta(i))$ and to check which of them satisfy the matching property.

From Proposition 5 we know the number of paths of $P_{2 n}$ passing through the point $(i, \eta(i))=\left(i, \frac{\epsilon(i)}{2}\right)$. These paths of $P_{2 n}$ can be determined by the subtree of the tree of $H_{2 n-1}$ presented in Section 2 .

For example, for $n=3$ and $\epsilon(4)=2$ the number of paths of $P_{6}$ passing through the point $(4,1)$ is equal to 2 . Using the corresponding subtree, we obtain the sequences 11111 and 12211 hence,

$$
\begin{aligned}
& W_{6}(4 ; 2)=W_{6}(222222) \cup W_{6}(244222)= \\
& =\{a \bar{b} b \bar{b} b \bar{a}, a \bar{b} b b \bar{b} \bar{a}, a b \bar{b} \bar{b} b \bar{a}, a b \bar{b} b \bar{b} \bar{a}\} \cup\{a a \bar{b} \bar{a} b \bar{a}, a a b \bar{a} \bar{b} \bar{a}\} \\
& =\{a \bar{b} b \bar{b} b \bar{a}, a \bar{b} b b \bar{b} \bar{a}, a b \bar{b} \bar{b} b \bar{a}, a b \bar{b} b \bar{b} \bar{a}, a a \bar{b} \bar{a} b \bar{a}, a a b \bar{a} \bar{b} \bar{a}\}
\end{aligned}
$$

Using the above algorithms, we have obtained the following enumeration results: A192927, A207851, A203019, A208357, A208358 in [10].

\section{References}

1. Delest, M.-P., Viennot, G.: Algebraic languages and polyominoes enumeration. Theoret. Comput. Sci. 34, 169-206 (1984)

2. Donaghey, R., Shapiro, L.W.: Motzkin numbers. J. Combin. Theory, Ser. A 23, 291-301 (1977)

3. Jiang, T., Lin, G.H., Ma, B., Zhang, K.: A general edit distance between RNA structures. Journal of Computational Biology 9(2), 371-388 (2002)

4. Lando, S., Zvonkin, A.: Plane and projective meanders. Theoret. Comput. Sci. 117, 227-241 (1993)

5. Nebel, M.E.: Combinatorial Properties of RNA Secondary Structures. Journal of Computational Biology 9, 541-573 (2001)

6. Panayotopoulos, A., Tsikouras, P.: The multimatching property of nested sets. Math. \& Sci. Hum. 149, 23-30 (2000)

7. Panayotopoulos, A., Tsikouras, P.: Properties of meanders. JCMCC 46, 181-190 (2003)

8. Panayotopoulos, A., Tsikouras, P.: Meanders and Motzkin words. J. Integer Seq. 7, Article 04.1.2 (2004)

9. Panayotopoulos, A., Vlamos, P.: Meandric polygons. Ars Combinatoria 87, 147-159 (2008)

10. Sloane, N.: The Online-Encyclopedia of Integer Sequences, published electronically at www.research.att.com/ njas/sequences/

11. Stein, P.R., Waterman, M.S.: On some new sequences generalizing the Catalan and Motzkin numbers. Discrete Math. 26, 261-272 (1978) 